\title{
La formación continua de los periodistas en RTVE: Especificidad tecnológica y puntos de encuentro con los perfiles y competencias profesionales en el Grado de Periodismo
}

The continuous training of journalists in RTVE: Specificity technological and professional profiles and competencies meeting points with a Journalism Degree

\author{
Miguel Angel Ortiz Sobrino \\ Profesor contratado Doctor Facultad de Ciencias de la Información \\ (Universidad Complutense de Madrid)
}

Fecha de recepción: 26 de noviembre de 2014

Fecha de revisión: 23 de enero de 2015

Para citar este artículo: Ortiz Sobrino, M.A. (2015): La formación continua de los periodistas en RTVE: Especificidad tecnológica y puntos de encuentro con los perfiles y competencias profesionales en el Grado de Periodismo, Icono 14, volumen (13), pp. 247-269. doi: 10.7195/ri14.v13i1.766 


\section{Resumen}

El presente estudio analiza los contenidos de la formación continua que reciben los Informadores de la Corporación Radiotelevisión Española (RTVE) y en qué medida inciden los cambios tecnológicos y organizativos en la formación para actualizar sus competencias profesionales. En ese sentido, se examinan los conocimientos y competencias demandadas como consecuencia de la incorporación de nuevas herramientas tecnológicas para la producción informativa y de las necesidades derivadas de planificación y organización de los recursos empresariales. El análisis se ha realizado a partir de los rasgos del perfil profesional del periodista recogido en el Libro Blanco de los actuales Títulos de Grado en Comunicación.

\section{Palabras clave}

Formación continua - RTVE - Competencias - Perfiles profesionales - Periodistas - Libro Blanco de los Grados en Comunicación

\section{Abstract}

This research analyzes the content of continuing training receive the journalists of Radio and Television Corporation (RTVE) and how much influence the technological and organizational changes in training to update their skills. In that sense, the knowledge and skills demanded because of the incorporation of new technological tools for information production and the needs arising from planning and organizing corporate resources are examined. The analysis was carried out using the features of the professional profile of journalists gathered in the White Paper on current Degrees in Communication.

\section{Key Words}

Continuous training - RTVE - Professional skill - Professional profiles - Journalists 


\section{Introducción}

El nuevo ecosistema de medios y el cambio de los procesos de producción han transformado los perfiles profesionales en las empresas de radio y televisión, sobre todo en la última década. A raíz de la aparición de las TIC y de los nuevos soportes de distribución de contenidos, la irrupción de innovadores formatos multimedia y las nuevas demandas de la audiencia han puesto de manifiesto la necesidad de plantear una formación continua de los profesionales de la información en las empresas audiovisuales. Sobre todo, a causa de la permanente transformación de la tecnología aplicada a la producción informativa (Tejedor, 2014; Pérez Tornero y Tejedor, 2014).

Aunque ha transcurrido relativamente poco tiempo desde que, en 2005, la Agencia Nacional de Evaluación de la Calidad y Acreditación (ANECA) publicó el Libro Blanco de los títulos de Grado en Comunicación, la definición de competencias y perfiles profesionales que se recogían en aquel texto ha quedado obsoleta con motivo de la aparición de nuevas herramientas digitales para la producción de contenidos y la consolidación de un panorama de convergencia mediática en constante transformación. En el caso del sector audiovisual, el Observatorio de Formación de Nuevas Tecnologías Audiovisuales (FONTA), de la Universidad Complutense de Madrid, ya ha advertido de que los cambios profundos a las que está siendo sometido el sector de la comunicación reclaman una redefinición de los perfiles profesionales para dotarlos de competencias acordes a las nuevas estrategias y soportes de la convergencia mediática. Para FONTA es imprescindible terminar con el divorcio universidad-empresa y garantizar la adaptación al mercado laboral de los futuros egresados de los Grados de Comunicación (Vivar et alt., 2010). En definitiva, un reciclaje profesional que debe ser contemplado como una revisión y actualización de ciertas premisas de las que se partía en el Libro Blanco, en aspectos relacionados con las competencias profesionales, las destrezas tecnológicas y los conocimientos de los comunicadores de la convergencia mediática (López García, 2010).

Esta obsolescencia de algunas de las características de los perfiles profesionales y la necesidad de actualizar el currículo formativo de los nuevos Graduados en Periodismo es lo que justifica la necesidad de implementar la formación continua

DOI: ri14.v13i1.766 | ISSN: 1697-8293 | Año 2015 Volumen 13 N¹ | ICONO14 
desde las empresas de Comunicación. En el trabajo que ahora se presenta, se toma como referencia el Libro Blanco de ANECA como marco para comprender en qué medida las empresas están propiciando la actualización de conocimientos y competencias de sus profesionales para adecuarlos a sus necesidades productivas.

Alguien podría pensar que esta necesidad de readaptación de los comunicadores al mercado laboral sólo afecta a los que pasaron por las Facultades de Comunicación hace años y a los profesionales de mayor antigüedad. Nada más lejos de la realidad. Una industria en constante transformación y sometida al impacto tecnológico, como sucede en el sector de la Comunicación, exige un continuo reciclaje profesional de sus trabajadores, incluidos los más jóvenes egresados de los títulos de Grado del Espacio Europeo de Educación Superior (EEES). En este sentido algunas organizaciones, como la Corporación Radiotelevisión Española (RTVE), vienen desarrollando una política de formación continua de sus periodistas para adaptar sus competencias y su perfil laboral a las nuevas necesidades productivas y tecnológicas del sector audiovisual (IRTVE,2007a; IRTVE, 2007b; IRTVE, 2013).

\section{Objetivos}

El objetivo principal del presente estudio se ha centrado en determinar los tipos de contenidos que los periodistas de la Corporación RTVE recibieron en los cuarenta y cinco cursos de formación continua impartidos por la propia empresa, en 2013, para adquirir competencias profesionales que les permitieran dominar el uso de las nuevas herramientas digitales de producción, mejorar la calidad de los contenidos informativos y hacer frente a las necesidades organizativas de la Corporación en aquel momento. No se trata de enumerar o describir minuciosamente los contenidos de estas acciones formativas, si no de establecer la tipología de formación requerida para la actualización de las competencias de este colectivo profesional, así como poner de manifiesto en qué medida determinados cambios organizativos o la necesidad de mejorar la calidad de los contenidos condicionan el reciclaje profesional. En ese sentido, y a efectos de una mayor fluidez del texto, sólo se mencionan en esta contribución aquellos que -a juicio de los responsables de la formación continua de

ICONO14 | Año 2015 Volumen $13 N^{\circ} 1$ | ISSN: 1697-8293 | DOI: ri14.v13i1.766 
RTVE- son los más relevantes y responden a una política de recursos humanos consensuada con los representantes de los agentes sociales en el seno de la Corporación (Verdugo, 2014; González Ladrón Guevara, 2014). No obstante, para las conclusiones y resultados que ahora se presentan, se ha tenido en cuenta el porcentaje de cursos y horas ofertadas en relación con la tipología de la formación, según los datos suministrados por el centro de formación de la Corporación (Álvarez Gómez, 2014; Puerto López, 2014). La hipótesis de partida se ha basado en el hecho de que los contenidos de esa formación continua tienen como objetivo primordial el reciclaje profesional de los periodistas y satisfacer las nuevas necesidades productivas de la empresa.

En paralelo al objetivo principal, el estudio persigue -también- conocer si esta formación empresarial tiene algunos puntos de encuentro con los conocimientos y competencias profesionales propias del perfil de periodista, recogidas en el Libro Blanco de los Títulos de Grado en Comunicación (ANECA, 2005:221).

Por último, de manera secundaria, interesa conocer qué perfiles profesionales de los contemplados en el texto de ANECA se corresponden con las diferentes categorías y perfiles de los actuales informadores que trabajan en la Corporación Radiotelevisión Española.

Los resultados de la investigación que más tarde se presentan recogen, en primer lugar, cuáles de los diferentes perfiles profesionales de los periodistas contemplados en el Libro Blanco están, a su vez, reflejados como perfiles o categorías profesionales en el actual Convenio Colectivo de esta corporación pública (B0E, 2014). A partir de ahí, se aborda especialmente la importancia de la adquisición de nuevas competencias tecnológicas y la tipología de formación requerida para adquirirla. Después, se repara en la importancia y el peso de la formación continua encaminada a la mejora de la calidad de los contenidos informativos. Finalmente, se refleja la percepción corporativa sobre la vigencia de los tres grupos de competencias contempladas en el Libro Blanco para los profesionales de los medios audiovisuales.

DOI: ri14.v13i1.766 | ISSN: 1697-8293 | Año 2015 Volumen 13 N¹ | ICONO14 


\subsection{Metodología y materiales}

Desde el punto de vista metodológico, se ha utilizado una técnica documental descriptiva reforzada con entrevistas en profundidad a los principales responsables de la formación continua de la radiotelevisión pública estatal. En ese sentido, el análisis ha partido de la lectura de algunas contribuciones científicas sobre la adaptación de los estudios de Periodismo al Espacio Europeo de Educación Superior, así como otra literatura científica y legal relacionada con las competencias y perfiles profesionales de los periodistas españoles en el sector audiovisual. Este procedimiento documental ha permitido contextualizar el trabajo de investigación y tomar las referencias científicas necesarias para enmarcar los resultados del análisis.

A continuación, se ha procedido a realizar un estudio pormenorizado de la oferta de formación continua llevada a cabo por el centro de formación de la Corporación RTVE para saber la capacitación profesional y el tipo de contenidos curriculares demandados desde sus áreas y departamentos de producción. El material utilizado para el estudio ha sido la Memoria anual del centro de formación de la radiotelevisión pública y la documentación complementaria sobre las necesidades formativas de la Corporación proporcionada por su departamento de formación (Álvarez Gómez, 2014).

Una vez obtenidos, los resultados del estudio se han relacionado con los aspectos y líneas que -desde esa perspectiva- se ponen de manifiesto en el Libro Blanco de los títulos de Grado en Comunicación promovido por ANECA (2005). En concreto, se ha partido del análisis del retrato robot del perfil del futuro periodista, sus competencias y la tipología de contenidos curriculares que debía cursar en el nuevo Grado de Periodismo, según el Libro Blanco. Seguidamente, se ha hecho una comparación con el Convenio Colectivo y el Plan de Formación de 2013 de la Corporación RTVE para establecer en qué medida se correspondían con la definición de la categoría profesional de "Informador" (periodista) que trabaja en la radiotelevisión pública estatal, con sus competencias y con la formación continua recibida. Después, se ha procedido a determinar cuales son los contenidos donde se da el mayor grado de especificidad de la formación continua de RTVE, en relación a los previstos en el Libro Blanco.

ICONO14 | Año 2015 Volumen 13 Nº 1 | ISSN: 1697-8293 | DOI: ri14.v13i1.766 
Finalmente, las entrevistas en profundidad al Director y Subdirector del centro de formación de la radiotelevisión pública estatal, así como a los dos responsables de coordinación y seguimiento de la implementación de la formación continua, han servido para verificar y reforzar los resultados obtenidos tras el trabajo de campo.

\section{Antecedentes y estado de la cuestión}

A efectos de la presentación de los resultados de la investigación que más adelante se ofrecen, es conveniente tomar en consideración un marco contextual en el que se incluyan los dos protagonistas principales del análisis y una revisión bibliográfica sobre el tema que nos ocupa.

\subsection{Contexto formativo y empresarial}

El marco del análisis realizado conviene situarlo desde la perspectiva de la enseñanza universitaria en España y desde la óptica de la formación continua empresarial en el sector público de radio y televisión.

En relación a los Grados de Comunicación en la Universidad española, Elena Real (2012) ha contabilizado en España treinta y siete centros universitarios en los que hoy se imparten los estudios de Periodismo. De ellos, veinte pertenecen a universidades públicas, quince son de universidades privadas, dos de la Iglesia Católica y tres corresponden a centros adscritos a universidades públicas. Tres años después, la sede electrónica del Ministerio de Educación (MEC, 2015) recoge la oferta de Grados en Periodismo de esas treinta y siete Universidades españolas. Según datos suministrados por la Asociación de la Prensa de Madrid, en el curso 2013/2014 habían veintiun mil cuatrocientos treinta y ocho alumnos matriculados en el Grado y Licenciaturas de Periodismo de las diferentes Universidades españolas (Palacio/ APM, 2014).

Conviene recordar que, traspasado el ecuador de la década pasada y de acuerdo al nuevo marco del Espacio Europeo de Educación Superior (EEES), muchas de las Facultades de Comunicación de esas universidades comenzaron a adaptar las an-

DOI: ri14.v13i1.766 | ISSN: 1697-8293 | Año 2015 Volumen 13 N¹ | ICONO14 
tiguas estructuras curriculares de las Licenciaturas de Comunicación en Títulos de Grado: Periodismo, Comunicación Audiovisual y Publicidad y Relaciones Públicas (del Olmo y Mora, 2010). Previamente, en 2005, la Agencia Oficial de Evaluación de Calidad y Acreditación (ANECA) promovió la elaboración de un Libro Blanco de esos títulos universitarios dónde se definían -de manera genérica- las competencias profesionales y los perfiles laborales que debían tener sus egresados, en concreto los futuros periodistas.

En cuanto a la formación continua impartida por la Corporación RTVE a sus empleados, conviene tomar como referencia la doble actividad de su centro corporativo de formación, el Instituto de RTVE: Por un lado, la impartición de cursos de formación continua; por otro, la realización de investigaciones y actividades relacionadas con los medios de comunicación audiovisuales -foros de debate, edición de publicaciones, etc.- que redundan habitualmente en la mejora de las competencias profesionales de los Informadores ( Ortiz Sobrino, Ruiz San Román y Díaz Cerveró, 2013).

La importancia de la formación continua como herramienta estratégica de la Corporación RTVE está recogida en el Capítulo IV -artículo 30- de su II Convenio Colectivo (BOE, 2014). En él se dice expresamente que la formación continua en la radiotelevisión pública está orientada, por un lado, a garantizar la formación adecuada a las necesidades de cada área de trabajo y, por otro, a actualizar los conocimientos profesionales exigibles en cada puesto funcional. Su diseño curricular responde a las necesidades formativas detectadas por los responsables de las diferentes unidades operativas de la empresa (IRTVE, 2013). De acuerdo a este texto legal, la formación continua se considera un derecho y, a la vez, un deber de los trabajadores, que están obligados a realizar un itinerario formativo anual diseñado por su centro de formación en el que los contenidos se centran especialmente en la innovación tecnológica y en la mejora de calidad de la producción (RTVE, 2007a; y RTVE, 2007b).

Tanto la actualización de conocimientos para afrontar los cambios organizativos y tecnológicos como el aprendizaje encaminado a la mejora de calidad de la producción informativa, tienen carácter obligatorio para los periodistas de la Corpora- 
ción RTVE. En torno a esos contenidos se elaboran los planes de formación continua anual, cuyo seguimiento y superación se computa como méritos en los planes de promoción de estos profesionales de la información (Ortiz Sobrino, 2013). Dada su consideración estratégica, la formación continua en la radio y la televisión estatal está supervisada por la Comisión General de Formación, integrada por representantes de la Corporación y delegados de los agentes sociales representados en su Comité de Empresa (González Ladrón Guevara, 2014).

\subsection{El perfil del Periodista en el Libro Blanco de los Títulos de Grado de Comunicación}

Como quiera que el objetivo del estudio se centró en la formación continua y adquisición de competencias profesionales de los periodistas de la radiotelevisión pública estatal, hubo que partir del perfil profesional del periodista y sus competencias, contemplado en el Libro Blanco de los Títulos de Grado en Comunicación (Periodismo), y determinar en qué medida esos perfiles estaban incorporados en la estructura actual de la Corporación RTVE.

\subsubsection{El periodista en el Libro Blanco de los títulos de Grado en Comunicación}

Se dice en este texto de ANECA (2005) que la titulación de Periodismo está diseñada a partir de cuatro perfiles profesionales básicos. En concreto, se citan los siguientes: Redactor de información periodística en cualquier tipo de soporte, Redactor o Responsable de prensa o comunicación institucional, Gestor de portales y editor de contenidos, e Investigador/docente y consultor de comunicación. En paralelo, en el Libro Blanco se hace referencia al perfil del profesional de la información que desarrolla su actividad impartiendo materias de comunicación en las enseñanzas superiores o medias.

Como Redactor de información periodística en cualquier tipo de soporte se considera en el Libro Blanco a aquel profesional del periodismo que desarrolla su actividad creando contenidos periodísticos en cualquier medio de comunicación electrónico o tradicional. A este perfil profesional se le asignan las tareas de redactor, reportero, presentador y director; también, el diseño, escritura y ejecución de guiones de reportajes y documentales audiovisuales o multimedia.

DOI: ri14.v13i1.766 | ISSN: 1697-8293 | Año 2015 Volumen 13 N¹ | ICONO14 
De igual forma, el texto de la ANECA identifica como Redactor o responsable de prensa y comunicación institucional a aquel periodista que ejerce sus funciones en el seno de un gabinete de prensa de una institución pública o privada, para coordinar y ejecutar los trabajos de carácter informativo o comunicativo.

Junto a estos dos perfiles, el Libro Blanco hace alusión a la figura del Gestor de portales y Editor de contenidos, identificándola con la del profesional de redacción y ejecución de trabajos de edición para empresas editoras o creadoras de producciones culturales e informativas. Se trata de especialistas en el tratamiento, la gestión y la edición de todo tipo de contenidos por medio de sistemas preferentemente digitales.

Finalmente, también se considera en el texto el perfil de Consultor de comunicación, cuyas competencias están encaminadas a las tareas de asesoría, consultoría y mediación en las empresas e instituciones.

\subsection{Una revisión de la literatura científica}

Los Grados de Comunicación, así como la orientación de los perfiles profesionales y las competencias de sus titulados, han sido abordados por diferentes autores y en publicaciones científicas monográficas sobre los estudios de Comunicación en el Espacio Europeo de Educación Superior (Marta Lazo, 2009; Sierra Sánchez y Cabezuelo Lorenzo, 2010; Vivar et alt., 2010; Agustín Lacruz, Marta Lazo y Ubieto Artur, 2014). En ellas se pueden encontrar contribuciones que enmarcan los nuevos Grados de Comunicación en el contexto de Bolonia (Real, 2009 y Ortiz Sobrino, 2009).

Otras contribuciones científicas se han hecho eco de las competencias y perfiles profesionales de los titulados en los diferentes Grados de Comunicación y su inserción en el mercado laboral (López Vidales y Ortiz Sobrino, 2011; De Vicente, 2011: Arroyo Cabello, 2011; Vinadert et alt., 2011; Vivar, 2011; Gobantes Bilbao, 2013; Flores Vivar, 2013; Marta Lazo y Ortiz Sobrino, 2013; Fanjul Peydró y González Oñate, 2013; Ortiz Sobrino (2013b); Sánchez Cid et al., 2014). En esta línea, también se puede hacer alusión a textos académicos españoles que han puesto de manifiesto la necesidad de actualizar la formación de los futuros comunicadores para adecuarlos a la convergencia de medios y el impacto de las TIC en la Comuni-

ICONO14 | Año 2015 Volumen $13 N^{\circ} 1$ | ISSN: 1697-8293 | DOI: ri14.v13i1.766 
cación (Campillo Alhama y Hernández Ruiz, 2010; Lorente, 2010; Pérez Tornero y Giraldo, 2010; Caerols y Viñarás, 2011; Rico Jerez ,2012; y Vázquez Ezcurdia, 2013; Tejedor, 2014;Pérez Tornero y Tejedor, 2014), así como otra literatura científica de autores extranjeros (Jenkins,2006 y 2010; Canavilhas y Satuf, 2013). Por su parte, la formación continua y los planes de carrera profesional en la Corporación RTVE han sido abordados por Ortiz Sobrino (2013a). Finalmente, en relación al Libro Blanco de los títulos de Grado en Comunicación en el que se recogen perfiles y competencias de los nuevos titulados, el propio texto de ANECA (2005) es la mejor referencia para contextualizar los actuales Grados en Comunicación y la formación continua de los profesionales de los media.

En todo caso, en relación con los perfiles profesionales en el sector audiovisual, esta literatura científica debe ser completada con otro tipo de documentos elaborados por consultoras, como $\mathrm{x}$, o con los propios convenios colectivos de las empresas y los convenios sectoriales específicos de la industria comunicativa en España.

De esta revisión bibliográfica se saca la conclusión de que, en relación al perfil dibujado por el Libro Blanco de ANECA, el perfil profesional y las competencias profesionales han ido transformándose de tal manera que ahora, en el sector audiovisual y en el resto de los sectores de la Comunicación, se están demandando profesionales más polivalentes y en un estado de formación permanente para adaptarse a la aparición de nuevas herramientas de producción. Hasta tal punto, que ya se habla de nuevos perfiles profesionales aparecidos como consecuencia de la irrupción de nuevos soportes digitales de producción y distribución de los contenidos: Perfiles y categorías profesionales que necesitan nuevas competencias, aunque mantengan otras que tradicionalmente han sido consustanciales al ejercicio de la profesión periodística.

\section{Resultados}

Terminado el análisis, se han obtenido resultados que ponen de manifiesto la incidencia de la tecnología en la formación competencial de los periodistas de la Corporación RTVE y cómo la mejora de la calidad de los contenidos requiere una formación continua de este colectivo profesional.

DOI: ri14.v13i1.766 | ISSN: 1697-8293 | Año 2015 Volumen 13 N 1 | ICONO14 


\subsection{La actual categoría laboral de Informador de la radiotelevisión pública se corresponde con algunos perfiles recogidos en el Libro Blanco de los Títulos de Comunicación}

Como antes se ha comentado, es conveniente enmarcar los resultados a partir de los diferentes perfiles de los periodistas recogidos en el Libro Blanco y en el actual Convenio Colectivo de RTVE.

De los cuatro perfiles de periodistas mencionados el texto de ANECA (2005), sólo tres tienen presencia profesional en la estructura de la radiotelevisión pública estatal (González Ladrón de Guevara, 2014; B0E, 2014). El Redactor de información periodística en cualquier tipo de soporte -identificado en RTVE con la categoría profesional de Informadores o Guionistas- desarrolla su actividad en los diferentes canales de Radio Nacional de España, Televisión Española y la web de la Corporación. Por su parte, los profesionales de la información que el Libro Blanco define como el Gestor de portales y editor de contenidos, prestan servicio fundamentalmente en el iRTVE: el portal de comunicación y repositorio de contenidos de la radiotelevisión pública estatal. Por último, la Dirección de Comunicación y Relaciones Institucionales acoge a los periodistas que responden al perfil de lo que el documento de la Agencia Nacional de Evaluación de la Calidad y Acreditación define como Responsable de Prensa o Comunicación Institucional.

\subsection{Tipología de los contenidos en la formación continua para los periodistas de RTVE}

Examinada la oferta de formación continua de 2013 en la Corporación RTVE, se puede observar -como se refleja en la siguiente Figura 1- que el número de acciones formativas dirigidas a sus periodistas estuvieron relacionadas, fundamentalmente, con la mejora de la calidad de los contenidos y la innovación tecnológica. En el caso de la capacitación técnica, la formación se llevó a cabo a través de cursos organizados desde la propia empresa (Innovación tecnológica) y mediante sesiones de formación implementadas por las empresas suministradoras de los nuevos equipos de producción (Expediente de compra). 


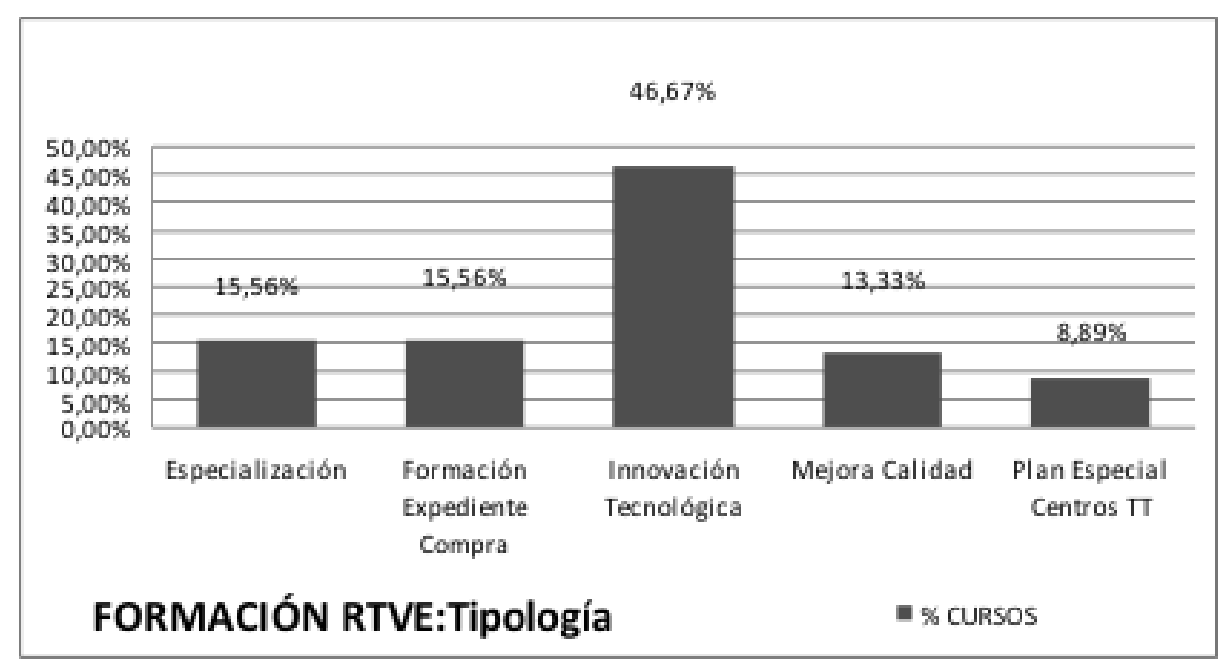

Figura 1: Fuente: elaboración propia sobre datos de la Memoria del IRTVE (Álvarez Gómez, 2014).

Sin embargo, si se toma como referencia el número de horas de formación -en lugar del número de cursos impartidos- los porcentajes cambian. En ese caso, se constata que el peso de la formación continua recibida por los periodistas se centró en contenidos dirigidos a la mejora de la calidad de la información, tal y como consta en la Figura 2.

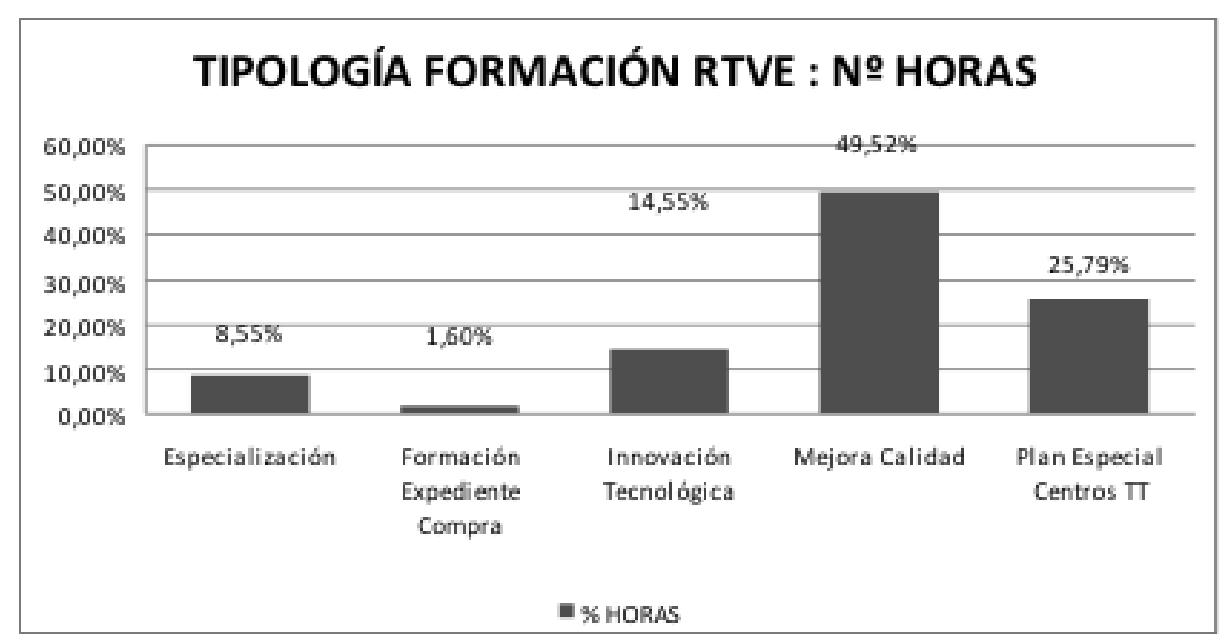

Figura 2: Fuente: elaboración propia sobre datos de la Memoria del IRTVE (2013). 


\subsection{La tecnología y la mejora de calidad de los contenidos informa- tivos son referentes imprescindibles para las nuevas competencias profesionales de la formación continua en la radiotelevisión pública}

Diez años después de realizado el diseño de las competencias de los futuros periodistas que fue recogido en el Libro Blanco de los Títulos de Grado en Comunicación, algunos de los principios que las sustentaban han quedado notoriamente obsoletas. De hecho, en su día algunos autores ya pusieron de manifiesto que el diseño de las competencias y perfiles profesionales contemplados en aquel texto no conseguía eliminar la barrera que tradicionalmente ha venido existiendo entre estos estudios universitarios y el mundo profesional (Marta Lazo et alt., 2012). De tal forma, que se puede constatar que hay una clara brecha entre la formación tecnológica contemplada en los estudios de Grado y los contenidos de la formación continua actual, motivada por el impacto de las TIC y la convergencia digital en el sector de la Comunicación (Jenkins,2006 y 2010; Canavilhas y Satuf, 2013). Especialmente en el sector audiovisual, ya que ahora el trabajo de los informadores es multicanal y requiere dominar el empaquetado y presentación de los contenidos para diferentes soportes de manera simultánea: pantalla de televisión, podscast de sonido, web, etc. (Verdugo, 2014).

Es en la capacitación tecnológica impartida en RTVE donde se producen las mayores diferencias respecto a las sugerencias que en ese sentido se hacen en el Libro Blanco, ya que su objetivo se centra en el dominio de herramientas digitales de última generación aplicadas a la producción informativa, no previstas en el momento de plantear las líneas generales de los nuevos Grados en Comunicación. Habitualmente, este tipo de acciones formativas pueden ser consecuencia de la incorporación del periodista a un nuevo puesto de trabajo dotado con una tecnología específica o bien estar motivada por la adquisición de nuevas herramientas digitales de producción informativa. En el primer caso, el responsable de la formación es el Instituto RTVE, el centro de formación de la Corporación, mientras que en el segundo es la propia empresa que suministra los equipos quien se encarga de formar a los trabajadores de la Corporación.

Como realidad específica y diferencial de la actualización de competencias profesionales de los periodistas en la radio y la televisión pública nacional, deben 
mencionarse, igualmente, los denominados "planes especiales de formación", que tienen un claro objetivo de reciclaje profesional y -por tanto- actualizan las competencias profesionales de los periodistas. Estos planes especiales se suelen implementar en situaciones de cambio organizativos para determinados centros de trabajo, como sucedió en 2013 con el Plan de Formación Especial para Centros Territoriales, cuando se unificaron los centros de producción territorial de Radio Nacional de España y Televisión Española.

De toda la formación continua dirigida a los periodistas de la Corporación RTVE, en 2013 destacaron especialmente los cursos cuyo objetivo era habilitar a los Informadores para trabajar en la producción de noticias sobre un entorno con tecnología Avid. Ese fue el caso de cursos como el de Operación iNews o el de Avid Newscutter para redacción digital, impartidos por su centro de formación. De igual manera, durante el pasado año fueron relevantes las acciones formativas sobre producción informativa con herramientas Avid llevados a cabo por las empresas que suministran esta tecnología a la Corporación RTVE. Entre ellos, el curso de Avid assist interplay, el de Avid command operation y el de Avid iNews Command en redacción digital. Estos contenidos se completaron con otras capacitaciones para la edición de noticias con otras herramientas digitales, como Edius y Instict (Verdugo, 2014).

En cuanto a los Informadores de Radio Nacional de España, lo más destacado fue la formación en las técnicas del sistema Dalet, utilizado en la producción informativa de la emisora pública, a través de dos cursos: uno sobre El Sistema Dalet + y otro sobre Dalet avanzado para informadores.

Durante este periodo, las acciones formativas para adaptar a los periodistas de la radiotelevisión pública a las exigencias de la convergencia mediática y a los nuevos escenarios informativos se centraron, especialmente, en contenidos relacionados con las redes sociales y el trabajo con sistemas digitales aplicados a la planificación informativa. Así sucedió con el curso sobre Diseño y comunicación a través de las redes sociales -orientado al aprendizaje de la gestión y coordinación de la actividad de los programas de radio y televisión en las redes sociales- y el curso Workflow digital, cuyo objetivo se basó en conocer el funcionamiento del flujo de trabajo en la redacción digital.

DOI: ri14.v13i1.766 | ISSN: 1697-8293 | Año 2015 Volumen 13 Nº 1 | ICONO14 
Como antes se ha comentado, a lo largo del pasado año se realizó una programación especial de cursos dirigida a los periodistas de los diferentes Centros Territoriales de la radiotelevisión pública para dar respuesta a las nuevas necesidades organizativas y tecnológicas de la empresa. Fundamentalmente, su objetivo se orientó a capacitar a los informadores en el manejo de las herramientas de edición de noticias Dalet e Inews, así como en las técnicas y sistemas de planificación informativa.

\subsection{La formación presencial para la mejora de la calidad informativa y los contenidos transversales en acciones formativas a distancia}

La formación continua presencial para la mejora de la calidad informativa estuvo constituida por cursos encaminados a la actualización y refuerzo de las capacidades productivas de los periodistas, en los que se acometió el aprendizaje de nuevas competencias para dominar las claves más actuales para elaborar la información audiovisual. A través de ellas, el periodista adquirió nuevas competencias relacionadas con la mejora de la calidad de la producción de contenidos, como el dominio de otros idiomas o la capacitación en técnicas de presentación de los contenidos informativos en radio y televisión.

Por su parte, la oferta de formación continua a distancia se fundamentó en contenidos relacionados, entre otros temas, con la producción televisiva, la elaboración de noticias o el lenguaje audiovisual. Su carácter transversal y genérico permitió que fuera seguida, de manera optativa, por todos los trabajadores de la radiotelevisión pública estatal, independientemente de la localización geográfica del centro de producción informativa al que estuviera adscrito (Álvarez Gómez, 2014 y Puerto López, 2014).

\subsection{La formación continua empresarial de la radiotelevisión pública incorpora ciertos conocimientos, aunque actualizados, que se ya se preveían en el Libro Blanco de los Títulos de Grado de Comunicación}

Tanto el Libro Blanco de los títulos de Grado en Comunicación como la formación continua de la radiotelevisión pública contemplan la necesidad de que los periodistas adquieran ciertos conocimientos y destrezas profesionales que afectan a los procesos de producción informativa o a la mejora de la calidad de los contenidos.

ICONO14 | Año 2015 Volumen $13 N^{\circ} 1$ | ISSN: 1697-8293 | DOI: ri14.v13i1.766 
Si se compara la tipología de competencias y conocimientos previstos en el Libro Blanco para los futuros periodistas salidos de los Grados de Comunicación con la formación continua empresarial recibida por este mismo colectivo profesional en la Corporación RTVE, se pueden observar ciertas similitudes aunque con algunas diferencias, al menos conceptualmente.

Ya el texto del Libro Blanco recomendaba que el periodista debía adquirir una serie de capacitaciones profesionales que facilitasen su inserción en el mercado laboral. En el caso del sector audiovisual, estas competencias profesionales pueden segmentarse en tres grupos: El primero hace referencia a la capacidad del informador para expresarse con fluidez y eficacia de manera oral, escrita 0 a través de imágenes en cada uno de los soportes de comunicación utilizados: radio, televisión y web; el segundo grupo de competencias se refiere al dominio de la tecnología para elaborar y transmitir la información; y, por último, el tercer grupo apunta tanto al dominio de las técnicas de selección de las fuentes, como a la capacidad de organizar la información y difundirla a través de cualquier soporte multimedia. En este sentido, cualquiera de estos tres grupos de competencias reflejadas en el Libro Blanco forman parte -también- de los objetivos de la formación continua empresarial de la radiotelevisión pública. Sin embargo, en el caso de la formación continua de la Corporación RTVE, los contenidos formativos para adquirir esos conocimientos y competencias profesionales están más actualizados y relacionados con las necesidades reales del quehacer informativo diario en las redacciones de los programas de radio y la televisión pública (Verdugo, 2014; González Ladrón Guevara, 2014).

\section{Discusión de los resultados}

Aunque la importancia de la formación y los itinerarios formativos obligatorios para los periodistas de RTVE es indiscutible y está recogida en su Convenio Colectivo, se detectan algunos aspectos de mejora en el planteamiento y diseño de los planes de formación continua dirigidos a los profesionales de la información de la radiotelevisión pública.

En concreto, del análisis realizado se observa que la formación transversal que se oferta a los periodistas de RTVE en la modalidad a distancia no se corresponde con

DOI: ri14.v13i1.766 | ISSN: 1697-8293 | Año 2015 Volumen 13 N¹ | ICONO14 
contenidos exclusivos para su perfil profesional. Al tratarse de una formación de carácter generalista, los cursos impartidos a través de la formación a distancia pueden ser estudiados, igualmente, por otros profesionales de esta la Corporación. A esta cuestión hay que añadir el hecho de que, aunque de acuerdo al Convenio Colectivo actual se demanda al periodista una serie de horas de formación anual para aumentar la calidad del trabajo y para adaptarse a los cambios tecnológicos y organizativos, lo más habitual es que sólo sea la formación tecnológica y la motivada por cambios organizativos la que tenga un carácter obligatorio. De tal forma, que en el caso de la formación a distancia puede ocurrir que, en algunas ocasiones, el periodista pueda elegir algunos de los contenidos en esta modalidad más por un interés personal que debido a un interés empresarial o de su mejora competencial: una apreciación compartida por los responsables de la formación de la Corporación (González Ladrón Guevara, 2014).

Se constata, igualmente, que los itinerarios formativos cursados por los periodistas a lo largo de los años son reconocidos por la Corporación y tienen consecuencias en su nivel retributivo, pero no obedecen a un plan concreto de carrera profesional individual de cada uno de ellos, tal y como se planteó en una propuesta realizada desde la propia empresa, en 2006, que de momento no ha sido implantada (Ortiz Sobrino, 2013 y Verdugo, 2014).

En consecuencia, parece conveniente que la formación continua atienda no sólo a la necesidad organizativa y tecnológica de la empresa, si no que tenga en cuenta también un auténtico plan de carrera del profesional de la información que le haga ganar competitividad y reconocimiento en el mercado laboral. Porque, precisamente, uno de los principales problemas ya detectados en el sector de la Comunicación es la certificación y validación de las competencias adquiridas fuera de la formación reglada (Vivar-Zurita y García García (2012).

\section{Conclusiones}

Los medios audiovisuales en el siglo XXI se han visto afectados por grandes cambios que han llegado de la mano de la digitalización y la convergencia mediática. Tanto una como otra, están afectando a todo el proceso productivo: desde la creación, hasta la posproducción y la difusión. Como consecuencia de este impacto

ICONO14 | Año 2015 Volumen 13 Nº 1 | ISSN: 1697-8293 | DOI: ri14.v13i1.766 
se han producido -y se seguirán produciendo- transformaciones importantes. Especialmente, en la producción y gestión de contenidos, en la forma de comunicar $\mathrm{y}$, por tanto, en los perfiles profesionales y sus competencias. Dice López García (2010) que los importantes cambios que se han producido en el hipersector de la Comunicación en los últimos veinte años han obligado a los periodistas conocer las nuevas herramientas de producción y renovar sus perfiles profesionales.

El uso de nuevas herramientas en la producción de noticias y la polivalencia profesional exigida por la convergencia mediática que posibilita la emisión de los contenidos a través de diferentes soportes, hacen absolutamente necesaria la adquisición de nuevas competencias acordes con el actual mercado productivo. No obstante, se percibe desde las empresas de comunicación que hay una formación genérica de los informadores adquirida en las Facultades de Comunicación que sigue estando vigente, independientemente de que, a veces, necesite ser reformulada.

Precisamente para redefinir y adaptar a los actuales periodistas a la permanente transformación del sistema productivo y tecnológico, las empresas de comunicación, como RTVE, vienen utilizando la formación continua.

En todo caso, y a tenor de los resultados obtenidos, todo indica que la redefinición de las competencias profesionales de los periodistas, exigida por un sector en constante transformación, les llevará a entrar en un proceso de formación continua y permanente durante toda su vida laboral.

\section{Referencias}

Agustín Lacruz, M. C., Marta Lazo, C. y Ubieto Artur, M. I. (Coord.) (2013). Perfiles profesionales y espacios de empleo en Información y Comunicación. Madrid: Icono14.

ANECA (2005). Libro Blanco de los títulos de Grado en Comunicación.

Arroyo Cabello, M. (2011). Aproximación al perfil del periodista en la posmodernidad. Razón y Palabra, 25, 1-11.

Recuperado de www.razonypalabra.org.mx/N/N76/varia/.../25_Arroyo_V76.pdf 
BOE(30 de enero 2014). II Convenio Colectivo de la Corporación RTVE.

Caerols, R. y Viñarás, M. (2011). Nuevas necesidades formativas para nuevos perfiles profesionales. En J. Sierra Sánchez y S. Liberal Ormaechea (ed.), Investigaciones educomunicativas en la sociedad multipantalla (pp. 667-697). Madrid: Fragua Campillo Alhama, C. y Hernández Ruiz A. (2010). Nuevas necesidades formativas para el perfil de gestor de eventos en el Grado de Publicidad y Relaciones Públicas. En J. Sierra Sánchez y F. Cabezuelo Lorenzo (coord.), Competencias y perfiles profesionales en los estudios de Ciencias de la Comunicación (pp.112134). Madrid: Fragua.

Canavilhas, J. \& Satuf, I. (2013). Jornalismo em transição: do papel para o tablet ... ao final da tarde. En Fidalgo, A. \& Canavilhas, J. (Ed.) Comunicação Digital: 10 anos de investigação (pp.35-60). Coimbra: Minerva.

Del Olmo Barbero, J. y Mora Roncero, A. (2010). Las competencias profesionales de los estudios de comunicación en el EEES: análisis del caso Aula Sur. La revista de alumnos de la URJC. En J. Sierra Sánchez y F. Cabezuelo Lorenzo (coord.), Competencias y perfiles profesionales en los estudios de Ciencias de la Comunicación (pp.220-239). Madrid: Fragua.

De Vicente, A.M. (2011). Nuevos perfiles laborales y docentes. Internet renueva el sector de la comunicación audiovisual. Telos, 87 Cuaderno Central, 1-8.

Fanjul Peyró, C. y González Oñate, C. (2013). Perfiles profesionales en Publicidad y Relaciones Públicas. En M. C. Agustín Lacruz; C. Marta Lazo; y M. I. Ubieto Artur (coord.), Perfiles profesionales y espacios de empleo en Información y Comunicación (pp.95-109). Madrid: Icono14.

Flores Vivar, J. M. (2013). Perfiles emergentes en la industria de la Comunicación on line. En M. C. Agustín Lacruz; C. Marta Lazo; y M. I. Ubieto Artur (coord.), Perfiles profesionales y espacios de empleo en Información y Comunicación (pp.31-53). Madrid: Icono14.

Gobantes Bilbao, M. (2013). El periodismo y sus perfiles profesionales en la prensa escrita. En M. C. Agustín Lacruz; C. Marta Lazo; y M. I. Ubieto Artur (coord.), Perfiles profesionales y espacios de empleo en Información y Comunicación (pp.53-71). Madrid: Icono14.

Jenkins, H. (2010), Piratas de textos. Fans, cultura participativa y televisión. Barcelona: Paidós.

Jenkins, H. (2006). Convergence Culture: Where Old and New Media Collid. N. York: 
University Press.

López García, X. (2010). La formación de los periodistas en el siglo XXI en Brasil,

España, Portugal y Puerto Rico. Revista Latina de Comunicación Social, 65, 231243. doi:10.4185/RLCS-65-2010-896-231-243

López Vidales, N. y Ortiz Sobrino, M.A. (2011). Viejas nomenclaturas, nuevas competencias. Perfiles profesionales en la radio española. Telos, 87 Dossier Central, 1-10.

Lorente, J. I. (2010). La formación de los comunicadores en el EEES: Hacia una nueva competencia comunicativa. En J. Sierra Sánchez y F. Cabezuelo Lorenzo (Coord.) Competencias y perfiles profesionales en los estudios de Ciencias de la Comunicación (pp.15-27). Madrid: Fragua

Marta Lazo, C. (2009). El proceso de reconversión de la Comunicación en el EEES. Icono14, 3, 1-7.

Marta Lazo, C.; Agustín Lacruz, M. C. y Ubieto Artur, M. I. (2012). Competencias interdisciplinares para la comunicación y la información en la sociedad digital. Madrid: Icono 14.

Marta Lazo, C. y Agustín Lacruz, M. C. (2013). Marco de referencia del mercado laboral de Información y Comunicación. En M. C. Agustín Lacruz; Marta Lazo, C.; y Ubieto Artur, M. I. (Coord.) Perfiles profesionales y espacios de empleo en Información y Comunicación (pp.141-161). Madrid: Icono14.

Marta Lazo, C. y Ortiz Sobrino, M. A. (2013). Gestión de los fondos documentales de Radio Nacional de España. El profesional de la Información 5, (22), 399-403. Ortiz Sobrino, M. A. (2012). La reconversión de la formación en comunicación desde el EEES. Icono14, 9, 35-49.

Ortiz Sobrino, M.A. (2013a). Le paradigme de classification et d'organisation des Ressources Humaines dans la radiotélévision publique espagnole:une proposition inachevée. Communication et Organisation, 43, 217-225.

Ortiz Sobrino, M.A. (2013b). Periodistas para la radio y la televisión de la convergencia multimedia: la puesta en valor de la polivalencia. En M. C. Agustín Lacruz; C. Marta Lazo; y M. I. Ubieto Artur (Coord.), Perfiles profesionales y espacios de empleo en Información y Comunicación (pp.71-95). Madrid: Icono14.

Ortiz Sobrino, M. A.; San Román Ruiz, J. A. y Díaz Cervero, E. (2013). Las televisiones y la investigación en Infancia y Televisión. Comunicar. Revista científica iberoamericana de comunicación y educación, 40, 137-166. doi:10.3916/C40- 
2013-03-04

Palacio, L./APM (2014). Informe anual de la profesión periodística. Madrid: Asociación de la Prensa de Madrid.

Pérez Tornero, J. M. y Giraldo, S. (2010). El ciberperiodismo en la Web 2.0: concepciones, perfiles y habilidades del periodista en la Red Social. En M. Cebrián Herreros (ed.), Desarrollos del periodismo por Internet (pp.17-40). Madrid: Comunicación Social.

Pérez Tornero, J. M. y Tejedor, S. (Dirs.). (2014). Escribir para la red. Reflexiones sobre la nueva (y vieja) escritura informativa online. Barcelona: Gabinete de Comunicación y Educación/UAB.

Real, E. (2009). Los estudios de Periodismo y el EEES ¿Avance o retroceso?. Icono14, $7(3), 8-34$.

Real, E. (2012). Los estudios de Periodismo en España, competencias y destrezas. En C. Marta Lazo; M. C. Agustín Lacruz y M. I. Ubieto Artur (Coord.), Competencias interdisciplinares para la comunicación y la información en la sociedad digital (pp. 115-145). Madrid: Icono 14.

RTVE (2007a). Memoria IRTVE.

RTVE (2007b). Líneas de trabajo para elaborar los itenierarios formativos en el nuevo sistema de clasifición profesional.

RTVE (2013). Memoria IRTVE.

Rico Jerez, M.(2012). Periodista multimedia interactivo: eje de los grupos de Comunicación españoles". Cuadernos de Información, 31, 103-116.

Sánchez Cid, M.; Benítez, A.; y San Martín Pascal, M. A. (2014). Formación y perspectiva laboral de los estudiantes de Ciencias de la Comunicación en el siglo XXI. Difícil, pero no imposible". En F. Ubierna Gómez y J. Sierra Sánchez (Coord.), Miscelánea sobre el entorno audiovisual en 2014 (pp. 601-619). Madrid: Fragua.

Tejedor,S. (2008). Ciberperiodismo y Universidad: diagnósticos y retos de la enseñanza del periodismo on-line. Análisi: Quaderns de comunicació i cultura. 36, 25-39.

Vázquez Ezcurdia, J. (2013). La profesión del Periodista: espacios y ámbitos para la formación. En M. C. Agustín Lacruz; C. Marta Lazo, C.; y M. I. Ubieto Artur (coord.), Perfiles profesionales y espacios de empleo en Información y Comunicación (pp. 253-261). Madrid: Icono14. 
Vinader, R.; Abuín, N. y García, A. (2011). Competencias digitales. El impacto web 2.0 en los perfiles profesionales del sector audiovisual. Telos, 87 Dossier Central, 1-11.

Vivar, H.; Abuín, N.; García, A.; Vinader, R. y Núñez, P. (2010). La transición al EEES: la especificidad como vía para incrementar la tasa de inserción laboral de los titulados en Comunicación. En J. Sierra Sánchez y F. Cabezuelo Lorenzo (Coord.), Competencias y perfiles profesionales en los estudios de Ciencias de la Comunicación (pp.27-44). Madrid: Fragua.

Vivar Zurita, H. (2011). TIC, Internet y el sector de la comunicación. Nuevos perfiles profesionales para una comunicación digital. Telos, 87 Dossier Central, 1-4.

Vivar-Zurita, H. y García-García, A. (2012). La adaptación de la formación reglada y continua a la Comunicación. Revista Latina de Comunicación Social, 67, 347361. doi: 10.4185/RLCS-067-959 / CrossRef link

\section{Entrevistas:}

- Jesús Verdugo Nieto, Director del Instituto RTVE.

- Eugenio González Ladrón de Guevara, Subdirector de Formación Externa Instituto RTVE.

- Julio Puerto López, Responsable de formación especializada.

- Andrés Alvarez Gómez, Responsable de Progamas Formación Contínua.

\section{Webgrafía:}

MEC (2015). Ministerio de Edución/sección títulos, https://www.educacion.gob.es/ ruct/consultaestudios?actual=estudios 